\title{
A KIRWAN BLOW-UP AND TREES OF VECTOR BUNDLES
}

\author{
G. TRAUTMANN
}

\begin{abstract}
In the paper [MTT] a conceptuel description of compactifications of moduli spaces of stable vector bundles on surfaces has been given, whose boundaries consist of vector bundles on trees of sufaces. In this article a typical basic case for the projective plane is described explicitly including the constrution of a relevant Kirwan blow up.
\end{abstract}

\section{INTRODUCTION}

To some extent, the replacement of limit sheaves in a compactification of a space of vector bundles by vector bundles on trees of surfaces is very natural, being in analogy to bubbling phenomena in Geometric Analysis and Yang-Mills theory in the work of Taubes, Uhlenbeck and Feehan. There the degeneration of connections and fields is described by a process where data are preserved by shifting them partially to a system of attached 4 -spheres. In the analogous situation of algebraic moduli spaces of vector bundles the attached 4 -spheres can be replaced by projective planes $\mathbb{P}_{2}$ hanged in at exceptional lines after blowing up points in a given surface. Then a limit sheaf can be transformed eventually to a vector bundle on the new reducible surface or on repeatedly constructed trees of surfaces. In [MTT] the trees of surfaces and vector bundles have been defined so that these objects can be the points of a compactification of the moduli spaces of rank-2 vector bundles on a given algebraic surface and are minimal for that purpose. The original basic example of such a compactification is the moduli space $M(2 ; 0,2)$ of stable rank-2 vector bundles with Chern classes $c_{1}=0, c_{2}=2$ on $\mathbb{P}_{2}$ which has partially been treated in [MTT]. In this paper an explicit construction of the Kirwan blow up of a relevant parameter space is given together with the construction of a universal family. In section 2 we recall shortly the definitions and the main theorem of [MTT] and in section 3 the typical limit trees are explicitly constructed.

Notation: All varieties in this article shall be defined over an algebraically closed field $k$ of characteristic zero. $P(V)$ denotes the projective space of lines in the $k$-vector space $V$, whereas $\mathbb{P}_{n}=P\left(k^{n+1}\right)$. The points of $P(V)$ are written as $[\mathrm{v}]$.

\section{TREES OF SURFACES AND BUNDLES}

\subsection{Trees.}

A tree $T$ in this article is a finite graph, oriented by a partial order $\leq$ and satisfying:

- there is a unique minimal vertex $\alpha \in T$, the $\operatorname{root}$ of $T$;

2010 Mathematics Subject Classification. 14J60, 14D06, 14D20, 14D23.

Key words and phrases. moduli, vector bundles, GIT-quotients, blowups. 
- for any $a \in T, a \neq \alpha$, there is a unique maximal vertex $b<a$, the predecessor of $a$, denoted by $a^{-}$;

- By $a^{+}:=\left\{b \in T \mid b^{-}=a\right\}$ we denote the set of direct successors of $a \in T$. We let $T_{t o p}$ denote the vertices of $T$ without successor.

A tree of surfaces over a given smooth projective surface $S$, modelled by a tree $T$, is a union

$$
S_{T}=S_{\alpha} \cup \bigcup_{a} S_{a}
$$

where

- $S_{\alpha}$ is a blow-up of $S$ in finitely many points

- for $a \in T_{t o p}, S_{a}$ is a projective plane $P_{a}=\mathbb{P}_{2}$

- if $\alpha \neq a \notin T_{t o p}, S_{a}$ is a blown up projective plane $P_{a}=\mathbb{P}_{2}$ in finitely many simple points not on a line $l_{a} \subset P_{a}$

- if $a \neq \alpha, S_{a} \cap S_{a^{-}}=l_{a}$ and $l_{a}$ is an exceptional line in $S_{a^{-}}$

Such trees can be construted by consecutive blow-ups of simple points, hanging in a $\mathbb{P}_{2}(k)$ in each exceptional line of the previous surface and then blowing up points in the new $\mathbb{P}_{2}$, the whole starting with the given surface $S$.

By the construction of $S_{T}$, all or a part of its components can be contracted. In particular, there is the morphism

$$
S_{T} \stackrel{\sigma}{\rightarrow} S
$$

which contracts all the components except $S_{\alpha}$ to the points of the blown up finite set of $S_{\alpha}$.

Note that:

1) There are no intersections of the components other than the lines $l_{a}$.

2) If $T=\{\alpha\}$ is trivial, then $S_{T}=S$.

3) After contracting the lines $l_{a}$ topologically (when defined over $\mathbb{C}$ ), one obtains bubbles of attached 4-spheres.

\subsection{Treelike vector bundles.}

A weighted tree is a pair $(T, c)$ of a tree $T$ with a map $c$ which assigns to each vertex $a \in T$ an integer $n_{a} \geq 0$, called the weight or charge of the vertex, subject to

$$
\# a^{+} \geq 2 \text { if } n_{a}=0 \text { and } a \neq \alpha .
$$

The total weight or total charge of a weighted tree is the sum $\Sigma_{a \in T} n_{a}=n$ of all the weights. We denote by $\mathbf{T}_{n}$ the set of all trees which admit a weighting of total charge $n$. It is obviously finite.

In the following we consider only pairs $\left(S_{T}, E_{T}\right)$, called $\mathbf{T}_{n}$-bundles or simply

tree bundles, where $T \in \mathbf{T}_{n}, S_{T}$ is a tree of surfaces and $E_{T}$ is a rank-2 vector bundle on $S_{T}$, such that $c_{1}\left(E_{T} \mid S_{a}\right)=0, c_{2}\left(E_{T} \mid S_{a}\right)=n_{a}$ for all weights $n_{a}$, and such that the bundles $E_{a}=E_{T} \mid S_{a}$ are "admissible", replacing a lacking stability condition, see [MTT].

In case $S_{T}=S$ this includes that the bundle $E$ on $S$ belongs to $M_{S, h}^{b}(2 ; 0, n)$, the quasiprojective Gieseker-Maruyama moduli scheme of $\chi$-stable rank-2 vector bundles on $S$ with respect to a polarization $h$ and of Chern classes $c_{1}=0, c_{2}=n$. The bundles in the special case of this article will all be admissible. 
In particular, an indecomposable bundle $E_{a}$ on $P_{a}=\mathbb{P}_{2}$ will be admissible if $c_{1}=0, c_{2}=1$. Such a bundle is not semistable on $P_{a}$. It is represented in homogeneous coordinates by exact sequences

$$
0 \rightarrow \mathcal{O}_{P_{a}}(-2) \stackrel{\left(z_{0}^{2}, z_{1}, z_{2}\right)}{\longrightarrow} \mathcal{O}_{P_{a}} \oplus 2 \mathcal{O}_{P_{a}}(-1) \rightarrow E_{a} \rightarrow 0 .
$$

We call the so defined tree bundles also $\mathbf{T}_{n}$-bundles. There is a natural notion of isomorphism for the pairs $\left(S_{T}, E_{T}\right)$. They consist of isomorphisms of the surfaces with the base surface fixed, and of isomorphisms of the lifted bundles.

\subsection{Families of tree bundles.}

A $\mathbf{T}_{n}$-family of tree bundles is a triple $(\mathbf{E} / \mathbf{X} / Y)$, where $\mathbf{X}$ is flat family of $\mathbf{T}_{n}$-surfaces $X_{y}, y \in Y$, and $\mathbf{E}$ is a rank-2 vector bundle on $\mathbf{X}$ such that each $E_{y}=\mathbf{E} \mid X_{y}$ is a $\mathbf{T}_{n}$-bundle.

One can then consider the moduli stack $\mathbb{M}_{n}$ defined by

$$
\mathbb{M}_{n}(Y):=\text { set of families }(\mathbf{E} / \mathbf{X} / Y)
$$

such that any bundle $E_{y}=\mathbf{E} \mid X_{y}$ is a 1-parameter limit of bundles in $M_{S, h}^{b}(2 ; 0, n)$. Let

$$
\mathbf{M}_{n}(Y)=\mathbb{M}_{n}(Y) / \sim
$$

be the associated functor. The following theorem is stated in [MTT].

Theorem: There is a separated algebraic space $M_{n}(S)$ of finite type over $k$ corepresenting the functor $\mathbf{M}_{n}$.

However the following questions are still open:

- Is $M_{n}(S)$ complete?

- When is $M_{n}(S)$ a (projective) scheme?

- Is $M_{n}\left(\mathbb{P}_{2}\right)$ a projective compactification of $M_{\mathbb{P}_{2}}(2 ; 0, n)$ ?

- Classification of limit tree bundles for $M_{\mathbb{P}_{2}}(2 ; 0, n)$ for $n \geq 3$ ?

- What about higher rank bundles on $\mathbb{P}_{2}$ ?

- Limit treelike bundles for instanton bundles on $\mathbb{P}_{3}$ ?

\section{Limit TREES FOR $M^{b}(0,2)$}

Let $M(2 ; 0,2)$ be the moduli space of semistable sheaves on $\mathbb{P}_{2}$ with Chern classes $c_{1}=$ $0, c_{2}=2$ and rank 2 and let $M^{b}(0,2)$ be its open part of (stable) bundles. It is well known that $M(2 ; 0,2)$ is isomorphic to the $\mathbb{P}_{5}$ of conics in the dual plane, the isomorphism being given by $[\mathcal{F}] \leftrightarrow C(\mathcal{F})$, where $[\mathcal{F}]$ is the isomorphism class of $\mathcal{F}$ and $C(\mathcal{F})$ is the conic of jumping lines of $[\mathcal{F}]$ in the dual plane.

It is also well known that any sheaf $\mathcal{F}$ from $M(2 ; 0,2)$ has two Beilinson resolutions on $P=\mathbb{P}_{2}=P(V)$

$$
\begin{gathered}
0 \rightarrow 2 \Omega_{P}^{2}(2) \stackrel{A}{\longrightarrow} 2 \Omega_{P}^{1}(1) \rightarrow \mathcal{F} \rightarrow 0 \\
0 \rightarrow 2 \mathcal{O}_{P}(-2) \stackrel{B}{\longrightarrow} 4 \mathcal{O}_{P}(-1) \rightarrow \mathcal{F} \rightarrow 0,
\end{gathered}
$$

where the matrices $A$ (of vectors in $V$ ) and $B$ (of vectors in $V^{*}$ ) are related by the exact sequence

$$
0 \rightarrow k^{2} \stackrel{A}{\longrightarrow} k^{2} \otimes V \stackrel{B}{\longrightarrow} k^{4} \rightarrow 0 .
$$


The conic $C(\mathcal{F})$ in the dual plane has the equation $\operatorname{det}(A)$.

$\mathcal{F}$ is locally free if and only if $C(\mathcal{F})$ is smooth or if and only if $\mathcal{F}$ is stable. If $C(\mathcal{F})$ decomposes into a pair of lines, then $A$ is equivalent to a matrix of the form $\left(\begin{array}{ll}x & 0 \\ z & y\end{array}\right)$, and then $\mathcal{F}$ is an extension

$$
0 \rightarrow \mathcal{I}_{[x]} \rightarrow \mathcal{F} \rightarrow \mathcal{I}_{[y]} \rightarrow 0
$$

whose extension class is represented by the entry $z$.

Notice here that the sheaf is still locally free at the point $[y]$ if the extension class is non-zero, i.e. $z \notin \operatorname{Span}(x, y)$. In any case $\mathcal{F}$ is $S$-equivalent to the direct sum $\mathcal{I}_{[x]} \oplus \mathcal{I}_{[y]}$.

\subsection{Type 1 degeneration}

In the following let $e_{0}, e_{1}, e_{2}$ be basis of $V$ and denote by $x_{0}, x_{1}, x_{2}$ its dual basis. For the first example, consider the 1-parameter deformation $\left(\begin{array}{cc}e_{0} & t a e_{1} \\ t b e_{2} & e_{0}\end{array}\right)$ with second Beilinson resolution

$$
\begin{gathered}
0 \rightarrow 2 \mathcal{O}_{C} \otimes \mathcal{O}_{P}(-2) \stackrel{B(t)}{\longrightarrow} 4 \mathcal{O}_{C} \otimes \mathcal{O}_{P}(-1) \rightarrow \mathbb{F} \rightarrow 0, \\
B(t)=\left(\begin{array}{cccc}
x_{1} & x_{2} & \operatorname{tax}_{0} & 0 \\
0 & t b x_{0} & x_{1} & x_{2}
\end{array}\right)
\end{gathered}
$$

with parameters $a, b$, where $C=\mathbb{A}^{1}(k)$. For $t=0$ the sheaf $\mathbb{F}_{0}$ is singular at $p=\left[e_{0}\right]$, $\mathbb{F}_{0}=\mathcal{I}_{p} \oplus \mathcal{I}_{p}$. The blowing-up $\sigma: Z \rightarrow C \times P$ at $(0, p)$ is the subvariety of $C \times P \times \mathbb{P}_{2}$ given by the equations

$$
t x_{0} u_{1}-x_{1} u_{0}=0, \quad t x_{0} u_{2}-x_{2} u_{0}=0, \quad x_{1} u_{2}-x_{2} u_{1}=0,
$$

where the $u_{\nu}$ are the coordinates of the third factor $\mathbb{P}_{2}$. We consider the following divisors on $Z$ :

- $\tilde{P}$, the proper transform of $\{0\} \times P$, isomorphic to the blow-up of $P$ at $p$;

- $D$, the exceptional divisor of $\sigma$;

- $H$, the lift of $C \times h$, where $h$ is a general line in $P$;

- $F$, the divisor defined by $\mathcal{O}_{Z}(F)=p r_{3}^{*} \mathcal{O}_{\mathbb{P}_{2}}(1)$,

as shown in the figure
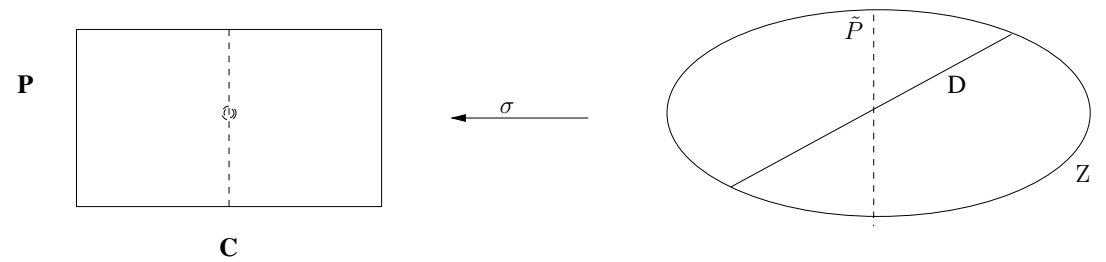

Then $D \sim H-F$, and we let $x_{\nu}$ resp. $u_{\nu}$ denote the sections of $\mathcal{O}_{X}(H) \operatorname{resp} . \mathcal{O}_{Z}(F)$ lifting the above coodinates. Using the equations of $Z$, we see that the canonical section $s$ of $\mathcal{O}_{Z}(D)$ is a divisor of the sections $x_{\nu}$, such that $t x_{0}=s u_{0}, x_{1}=s u_{1}, x_{2}=s u_{2}$ and 
gives rise to the diagram

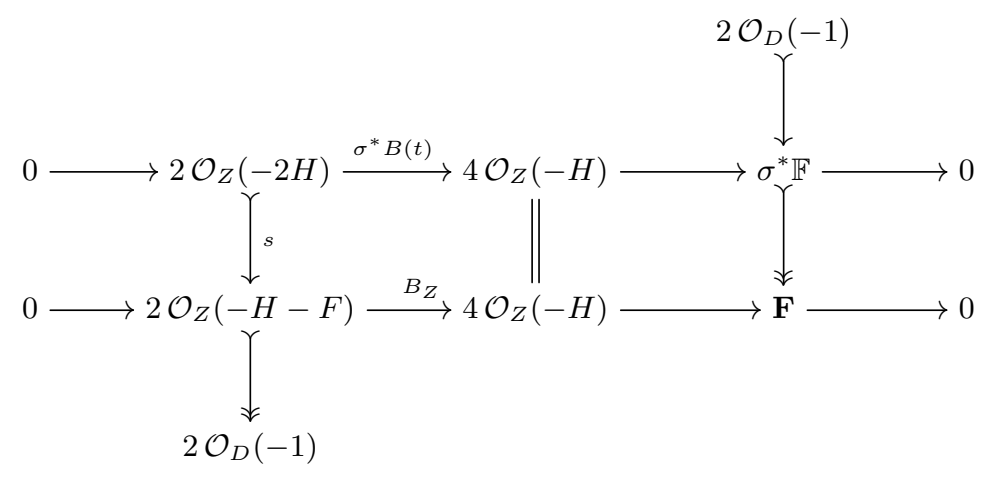

with $B_{Z}=\left(\begin{array}{cccc}u_{1} & u_{2} & a u_{0} & 0 \\ 0 & b u_{0} & u_{1} & u_{2}\end{array}\right)$. Thus $B_{Z}$ represents a locally free sheaf $\mathbf{F}$ on $Z$, but its first Chern class has been modified by blowing up and removing the torsion. To correct this, consider the twisted bundle $\mathbf{E}:=\mathbf{F}(D)$. Then $\mathbf{E} \mid \tilde{P} \simeq 2 \mathcal{O}_{\tilde{P}}$, and the restriction $\mathbf{E} \mid D$ belongs to $M_{D}^{b}(2 ; 0,2), D \simeq \mathbb{P}_{2}$. Moreover, $Z$ is flat over $C$ and $\mathbf{E}$ is a flat family of vector bundles over $C$ with the limit tree bundle $\mathbf{E} \mid Z_{0}$ on the fibre $Z_{0}=\tilde{P} \cup D$ over $0 \in C$. This can be symbolized by

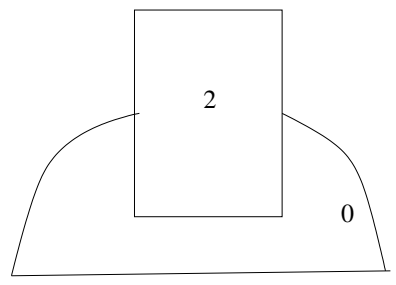

type 1

the numbers indicating the second Chern classes of the bundles on the components. The isomorphism class of this limit depends on the chosen parameters $a, b$ which determine a normal direction to the Veronese surface in $\mathbb{P}_{5}$. This leads to blowing it up and to the Kirwan blow-up of the parameter space, see section 4 .

\subsection{Type 2 degeneration}

Let now a family on $C \times P$ be given by $\left(\begin{array}{cc}e_{0} & -t e_{1} \\ -t e_{1} & e_{2}\end{array}\right)$, defining a deformation of the sheaf of $\left(\begin{array}{cc}e_{0} & 0 \\ 0 & e_{2}\end{array}\right)$. Similarly to the previous case, the deforming sheaf $\mathbb{F}$ is the cokernel of the matrix

$$
B(t)=\left(\begin{array}{cccc}
x_{2} & x_{1} & t x_{0} & 0 \\
0 & t x_{2} & x_{1} & x_{0}
\end{array}\right)
$$

Blowing up $C \times P$ in the two singular points $\left(0, p_{0}\right)$ and $\left(0, p_{2}\right), p_{\nu}=\left[e_{\nu}\right]$, leads to the figure
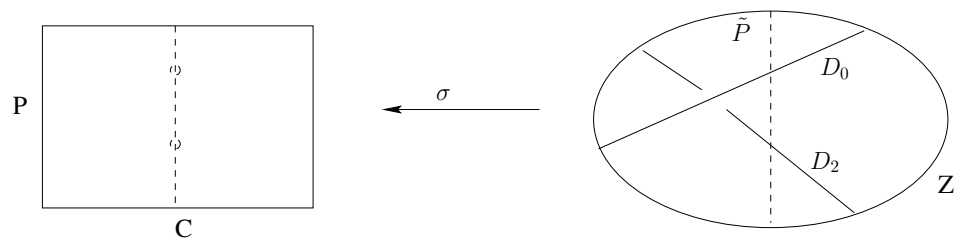

The blown up variety $Z$ has the standard embedding into $(C \times P) \times \mathbb{P}_{2} \times \mathbb{P}_{2}$ with divisors 
- $H$, the pull back of the divisor $C \times h$ in $C \times P$

- $\tilde{P}$, the blow-up of $\{0\} \times P$ in the two points

- $D_{0}, D_{2}$, the two exceptional divisors

- $F_{0}, F_{2}$, whose invertible sheaves are the pull backs of $\mathcal{O}_{\mathbb{P}_{2}}(1)$ from the third and fourth factor.

Letting denote $x_{\nu}, u_{\nu}, v_{\nu}$, and $s_{0}, s_{2}$ the basic sections of the sheaves of $H, F_{0}, F_{2}, D_{0}, D_{2}$, we have the equations (as homomorphisms between invertible sheaves) $t x_{0}=s_{0} u_{0}, \quad x_{1}=$ $s_{0} u_{1}, \quad x_{2}=s_{0} u_{2}$, and $x_{0}=s_{2} v_{0}, \quad x_{1}=s_{2} v_{1}, \quad t x_{2}=s_{2} v_{2}$.

By that we have the matrix decomposition

$$
\left(\begin{array}{cccc}
x_{2} & x_{1} & t x_{0} & 0 \\
0 & t x_{2} & x_{1} & x_{0}
\end{array}\right)\left(\begin{array}{cc}
s_{0} & 0 \\
0 & s_{2}
\end{array}\right)=\left(\begin{array}{cccc}
u_{2} & u_{1} & u_{0} & 0 \\
0 & v_{2} & v_{1} & v_{0}
\end{array}\right) .
$$

Using this, the torsion of $\sigma^{*} \mathbb{F}$ can be removed as in the diagram of the previous section. Then $\mathbf{F}=\sigma^{*} \mathbb{F} /$ torsion has the resolution

$$
0 \rightarrow \mathcal{O}_{Z}\left(-H-F_{0}\right) \oplus \mathcal{O}_{Z}\left(-H-F_{2}\right) \stackrel{B_{Z}}{\longrightarrow} 4 \mathcal{O}_{Z}(-H) \rightarrow \mathbf{F} \rightarrow 0,
$$

where $B_{Z}$ is the right hand matrix. The tree components of $\mathbf{F}$ are $\mathbf{F} \mid D_{i}=\mathcal{T}_{D_{i}}(-1)$, $\mathbf{F} \mid \tilde{P}=\mathcal{O}_{\tilde{P}}\left(-l_{0}\right) \oplus \mathcal{O}_{\tilde{P}}\left(-l_{2}\right)$, where $l_{0}, l_{2}$ are the exceptional lines on $\tilde{P}$. However, there is no way by twist or elementary transformation to make the first Chern classes $c_{1}$ vanish.

But starting with $\left(\begin{array}{cc}e_{0} & -t^{2} e_{1} \\ -t^{2} e_{1} & e_{2}\end{array}\right)$, we get by the same procedure a sheaf $\mathbf{F}$ on $Z$ whose resolution matrix is

$$
B_{Z}=\left(\begin{array}{cccc}
u_{2} & u_{1} & t u_{0} & 0 \\
0 & t v_{2} & v_{1} & v_{0}
\end{array}\right) .
$$

This resolution implies that $\mathbf{F}$ is reflexive and singular in exactly two points $q_{0}=\left\{u_{1}=\right.$ $\left.u_{2}=t=0\right\}$ and $q_{2}=\left\{v_{1}=v_{0}=t=0\right\}$, and that its restrictions to the components of $Z_{0}=\tilde{P} \cup D_{0} \cup D_{2}$ are $\mathbf{F} \mid \tilde{P}=\mathcal{O}_{\tilde{P}}\left(-l_{0}\right) \oplus \mathcal{O}_{\tilde{P}}\left(-l_{2}\right)$ and $\mathbf{F} \mid D_{i}=\mathcal{O}_{D_{i}} \oplus \mathcal{I}_{q_{i}, D_{i}}(1)$.

Hence there is an elementary transform on $\mathrm{Z}$,

$$
0 \rightarrow \mathbf{F}^{\prime} \rightarrow \mathbf{F} \rightarrow \mathcal{O}_{D_{0}} \oplus \mathcal{O}_{D_{2}} \rightarrow 0 .
$$

The resolution of $\mathbf{F}^{\prime}$ can be computed as follows. There is a decomposition $t u_{0}=s_{0} \tilde{u}_{0}$ because $t u_{0}$ vanishes on the divisor $D_{0}$. Similarly we have $t v_{2}=s_{2} \tilde{v_{2}}, u_{1}=s_{2} \overline{u_{1}}, v_{1}=$ $s_{0} \bar{v}_{1}$, and from this the matrix decomposition

$$
\left(\begin{array}{cccc}
u_{2} & \overline{u_{1}} & \tilde{u}_{0} & 0 \\
0 & \tilde{v_{2}} & \overline{v_{1}} & v_{0}
\end{array}\right)\left(\begin{array}{ccc}
1 & & \\
& s_{2} & \\
& & s_{0} \\
& & 1
\end{array}\right)=\left(\begin{array}{cccc}
u_{2} & u_{1} & t u_{0} & 0 \\
0 & t v_{2} & v_{1} & v_{0}
\end{array}\right)
$$

It follows by diagram chasing that the left hand matrix gives the resolution

$$
0 \rightarrow \mathcal{E}_{1} \rightarrow \mathcal{E}_{0} \rightarrow \mathbf{F}^{\prime} \rightarrow 0
$$

where $\mathcal{E}_{1}=\mathcal{O}_{Z}\left(-H-F_{0}\right) \oplus \mathcal{O}_{Z}\left(-H-F_{2}\right)$ and $\mathcal{E}_{0}=\mathcal{O}_{Z}(-H) \oplus \mathcal{O}_{Z}\left(-H-S_{2}\right) \oplus \mathcal{O}_{Z}\left(-H-S_{0}\right) \oplus \mathcal{O}_{Z}(-H)$ 
This resolution shows that $\mathbf{F}^{\prime}$ is locally free on $Z$. In order to determine its restrictions to the components, one should use the identities $u_{0}^{2}=x_{0} \tilde{u_{0}}, v_{2}^{2}=x_{2} \tilde{v_{2}}$, which follow from the previous identities. Using these, one can determine the restrictions of the twisted bundle $\mathbf{E}:=\mathbf{F}^{\prime}\left(D_{0}+D_{2}\right):$

$\mathbf{E} \mid \tilde{P}=2 \mathcal{O}_{\tilde{P}}$ and $\mathbf{E} \mid D_{i}$ is a bundle on $D_{i} \cong \mathbb{P}_{2}$ with Chern classes $c_{1}=0, c_{2}=1$ (see the description of bundles with these Chern classes in 2.2.)

Since the elementary transform and the twisting do not affect the bundle on the part of $Z$ over $C \backslash\{0\}$, the sheaf is a limit tree bundle on the fibre $Z_{0}=\tilde{P} \cup D_{0} \cup D_{2}$.

\subsection{Type 3 degeneration}

Let $\tilde{\mathbb{P}}_{5}$ be the blow-up of $P\left(S^{2} V\right)=\mathbb{P}_{5}$ of the Veronese surface in $\mathbb{P}_{5}$, let $\Sigma_{2} \subset \tilde{\mathbb{P}}_{5}$ be the exceptional divisor and $\Sigma_{1} \subset \tilde{\mathbb{P}}_{5}$ the proper transform of the divisor of degenerate conics, see also 4.5 .

By the above, type 1 limit tree bundles belong to $\Sigma_{2} \backslash \Sigma_{1}$ and type 2 limit tree bundles belong to $\Sigma_{1} \backslash \Sigma_{2}$. There is a third type of limit tree bundle belonging to $\Sigma_{2} \cap \Sigma_{1}$ with symbolic tree

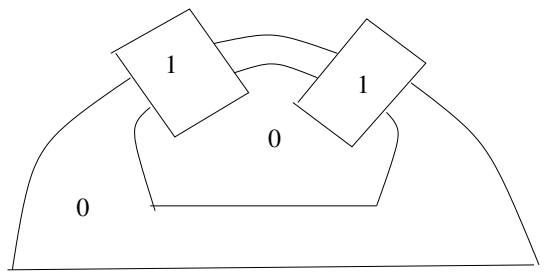

Examples can be obtained as limits of families of type $\left(\begin{array}{cc}e_{0} & -t^{3} e_{1} \\ -t^{3} e_{1} & e_{0}+t e_{2}\end{array}\right)$ and two consecutive blow-ups. In this case the family $\mathbb{F}$ on $C \times P$ is given as the cokernel in

$$
\begin{gathered}
0 \rightarrow 2 \mathcal{O}_{C} \otimes \mathcal{O}_{P}(-2) \stackrel{B(t)}{\longrightarrow} 4 \mathcal{O}_{C} \otimes \mathcal{O}_{P}(-1) \rightarrow \mathbb{F} \rightarrow 0, \\
B(t)=\left(\begin{array}{cccc}
x_{2} & x_{1} & t^{3} x_{0} & 0 \\
0 & t^{2} x_{2} & x_{1} & t x_{0}-x_{2}
\end{array}\right) .
\end{gathered}
$$

This sheaf $\mathbb{F}$ is singular in $(0, p), p=\left[e_{0}\right]$. Let then

$$
\sigma: Z \rightarrow C \times P
$$

be the blow-up as in 3.1, described as subvariety of $Z \subset C \times P \times \mathbb{P}_{2}$ with divisors $\tilde{P}, H, D, F, D \sim H-F$. Let $s$ be the standard section of $\mathcal{O}_{Z}(D)$ for the exceptional divisor, and let $x_{\nu}$ respectively $y_{\nu}$ be the basic sections of $\mathcal{O}_{Z}(H)$ respectively $\mathcal{O}_{Z}(F)$ with equations $t x_{0}=s y_{0}, x_{1}=s y_{1}, x_{2}=s y_{2}$. It follows as in 3.1 that the sheaf $\mathbf{F}=$ $\sigma^{*} \mathbb{F} /$ tor sion has the resolution

$$
\begin{gathered}
0 \rightarrow 2 \mathcal{O}_{Z}(-H-F) \stackrel{B_{Z}}{\rightarrow} 4 \mathcal{O}_{Z}(-H) \rightarrow \mathbf{F} \rightarrow 0, \\
B_{Z}=\left(\begin{array}{cccc}
y_{2} & y_{1} & t^{2} y_{0} & 0 \\
0 & t^{2} y_{2} & y_{1} & y_{0}-y_{2}
\end{array}\right) .
\end{gathered}
$$

This sheaf and its syzygy is of the same type as in 3.2 . It is reflexive and singular exactly in the points $p_{0}, p_{2} \in D \backslash \tilde{P}, \quad p_{0}=\left\{t=y_{1}=y_{2}=0\right\}$ and $p_{2}=\left\{t=y_{1}=y_{0}-y_{2}=0\right\}$. Againe one can verify that the sheaf $\mathbf{F}^{\prime}:=\mathbf{F}(D)$ has the restictions

$$
\mathbf{F}^{\prime} \mid \tilde{P}=2 \mathcal{O}_{\tilde{P}} \quad \text { and } \quad \mathbf{F}^{\prime} \mid D=\mathcal{I}_{p_{0}, D} \oplus \mathcal{I}_{p_{2}, D}
$$

on the components of $Z_{0}=\tilde{P} \cup D$. 
In order to construct a locally free limit tree bundle we blow-up $Z$ in the two points $p_{0}, p_{2}$ to get

$$
\tau: W \rightarrow Z
$$

with exceptional divisors $S_{0}$ and $S_{2}$, the proper transform $\tilde{D}$ of $D$, the lifted divisors $\tilde{P}$ and $F$, and the two divisors $F_{0}$ and $F_{2}$ coming from the embedding.

As in 3.2 one concludes that the sheaf $\mathbf{F}^{\prime \prime}=\tau^{*} \mathbf{F}^{\prime} /$ tor sion is reflexive and the cokernel of a matrix

$$
B_{Z}=\left(\begin{array}{cccc}
u_{2} & u_{1} & t u_{0} & 0 \\
0 & t v_{2} & v_{1} & v_{0}
\end{array}\right)
$$

and such that $\mathbf{F}^{\prime \prime}$ restricts as

$$
\mathbf{F}^{\prime \prime}\left|\tilde{P}=2 \mathcal{O}_{\tilde{P}}, \quad \mathbf{F}^{\prime \prime}\right| \tilde{D}=\mathcal{O}_{\tilde{D}}\left(-l_{0}\right) \oplus \mathcal{O}_{\tilde{D}}\left(-l_{2}\right), \quad \mathbf{F}^{\prime \prime} \mid S_{i}=\mathcal{O}_{S_{i}} \oplus \mathcal{I}_{q_{0}, S_{i}}(1),
$$

where $q_{i} \in S_{i} \backslash \tilde{D}$.

Finally, as in 3.2 , there is an elementary transform

$$
0 \rightarrow \mathbf{E}^{\prime} \rightarrow \mathbf{F}^{\prime \prime} \rightarrow \mathcal{O}_{S_{0}} \oplus \mathcal{O}_{S_{0}}
$$

such that $\mathbf{E}^{\prime}$ is locally free on $W$ and such that $\mathbf{E}:=\mathbf{E}^{\prime}\left(S_{0}+S_{2}\right)$ has the desired restrictions

$$
\mathbf{E}\left|\tilde{P}=2 \mathcal{O}_{\tilde{P}}, \quad \mathbf{E}\right| \tilde{D}=2 \mathcal{O}_{\tilde{D}},
$$

and such that $\mathbf{E} \mid S_{i}$ do have the Chern classe $c_{1}=0, c_{2}=1$. So $\mathbf{E}$ is a limit tree bundle on the tree of surfaces $W_{0}=\tilde{P} \cup \tilde{D} \cup S_{0} \cup S_{2}$.

\section{KIRWAN BLOW-UP I}

The $2 \times 2$-matrices with entries in $V$ in (1) parametrize the sheaves in $M(2 ; 0,2)$ and at the same time the conics of their jumping lines in the dual plane $P\left(V^{*}\right)$ by their determinants in $S^{2} V$. Since the isomorphisms of the left hand term in (1) are not essential, only the subspaces $[A]$ spanned by the rows of $A$ matter, so that the Grassmannian $G_{2}\left(k^{2} \otimes V\right)$ is a parameter space of $M(2 ; 0,2)$. The Plücker embedding

$$
p: G_{2}\left(k^{2} \otimes V\right) \subset P\left(\wedge^{2}\left(k^{2} \otimes V\right)\right)=P\left(\wedge^{2} k^{2} \otimes S V \oplus S^{2} k^{2} \otimes \wedge^{2} V\right)
$$

can be expressed in terms of the entries, using the standard basis of $k^{2}$, by

$$
\left[\left(\begin{array}{ll}
x & x^{\prime} \\
y & y^{\prime}
\end{array}\right)\right] \quad \stackrel{p}{\mapsto} \quad\left[x y^{\prime}-x^{\prime} y ; x \wedge y, x \wedge y^{\prime}+x^{\prime} \wedge y, x^{\prime} \wedge y^{\prime}\right] .
$$

One should note here that there is the relation

$$
\left(\begin{array}{ll}
x & x^{\prime} \\
y & y^{\prime}
\end{array}\right) \wedge\left(\begin{array}{cccc}
x \wedge y & x \wedge y^{\prime}+x^{\prime} \wedge y & x^{\prime} \wedge y^{\prime} & 0 \\
0 & x \wedge y & x \wedge y^{\prime}+x^{\prime} \wedge y & x^{\prime} \wedge y^{\prime}
\end{array}\right)=0 .
$$

There is an action of $\mathrm{SL}_{2}(k)$ on both sides of the Plücker embedding, induced by the natural action on $k^{2}$ and written as

$$
[A] g=[A g] \quad \text { and } \quad[q ; \Phi] g=\left[q ; \Phi S^{2} g\right]
$$


explicitly with

$$
A g=\left(\begin{array}{ll}
x & x^{\prime} \\
y & y^{\prime}
\end{array}\right)\left(\begin{array}{ll}
\alpha & \beta \\
\gamma & \delta
\end{array}\right) \quad \text { and } \Phi S^{2} g=(\xi, \omega, \eta)\left(\begin{array}{ccc}
\alpha^{2} & 2 \alpha \beta & \beta^{2} \\
2 \alpha \gamma & \alpha \delta+\beta \gamma & 2 \beta \delta \\
\gamma^{2} & 2 \gamma \delta & \delta^{2}
\end{array}\right)
$$

such that the Plücker embedding is equivariant. An element $[\mathrm{A}]$ in the Grassmannian is semistable if and only if $\operatorname{det}(A) \neq 0$, and it is stable if and only if $\operatorname{det}(A)$ is the equation of a non-degenerate quadric in the dual plane $P\left(V^{*}\right)$. Moreover, the morphism $[A] \rightarrow[\operatorname{det}(A)]$

$$
G_{2}\left(k^{2} \otimes V\right)^{s s} \longrightarrow P S^{2} V \cong \mathbb{P}_{5} \cong \bar{M}(2 ; 0,2,0)
$$

is a good GIT quotient, see [NT].

For the construction of a compactification of $M^{b}(0,2)$ by tree bundles we need to replace the Grassmannian by a parameter space with only stable points in order to avoid unnatural identifications in the boundary. This is done by the method of F. Kirwan, Ki] in two consecutive blow-ups.

The first blow-up: In the following we use the abbreviations $X=G_{2}\left(k^{2} \otimes V\right)$ and $G=\mathrm{SL}_{2}(k)$. The group $G$ has the fixed points $\left[\left(\begin{array}{cc}x & 0 \\ 0 & x\end{array}\right)\right]$. According to $[\mathrm{Ki}]$, let then $Z_{G}$ denote the subset

$$
Z_{G}=\{[A] \in X \mid \text { the affine fibre of } p(A) \text { fixed by } G\} .
$$

It follows that

$$
Z_{G}=\left\{\left[\left(\begin{array}{ll}
x & 0 \\
0 & x
\end{array}\right)\right]\right\} \cong P(V),
$$

that it is a closed and smooth subvariety of $X$ and that $Z_{G}=G Z_{G}$. The vanishing of the components of $\Phi$ characterizes the points of $Z_{G}$ and these components define its ideal sheaf $\mathcal{I}_{G}$. Let then

$$
\tilde{X}:=B l_{Z_{G}}(X)
$$

be the blow-up of $X$ along $Z_{G}$. In this situation

$$
\tilde{X} \subset X \times P\left(S^{2} k^{2} \otimes \wedge^{2} V\right)
$$

is the closure of the graph of the map $\Phi: X \backslash Z_{G} \rightarrow P\left(S^{2} k^{2} \otimes \wedge^{2} V\right)$. This blow-up can geometrically be described as follows.

4.1. Lemma: (a) $\tilde{X}$ is the subvariety of $X \times P\left(S^{2} k^{2} \otimes \wedge^{2} V\right)$ of points $([A],[\xi, \omega, \eta])$ satisfying

(i) $\left(x \wedge y, x \wedge y^{\prime}+x^{\prime} \wedge y, x^{\prime} \wedge y^{\prime}\right) \in k(\xi, \omega, \eta)$

(ii) $A \wedge\left(\begin{array}{cccc}\xi & \omega & \eta & 0 \\ 0 & \xi & \omega & \eta\end{array}\right)=0$

(b) The exceptional divisor $E_{G}$ in $\tilde{X}$ is the subvariety of pairs $([A],[x \wedge u, x \wedge w, x \wedge v])$ with $A=\left(\begin{array}{cc}x & 0 \\ 0 & x\end{array}\right)$.

(c) $\tilde{X}$ is smooth and the projection $\tilde{X} \rightarrow X$ is G-equivariant.

Sketch of proof: Because $\tilde{X}$ is the closure of graph, (i) follows immediately, and also (ii) by formula (2). Let conversely $Y \subset X \times P\left(S^{2} k^{2} \otimes \wedge^{2} V\right)$ be defined by (i) and (ii). Then $\tilde{X} \subset Y$ and $\tilde{X} \backslash E_{G}=Y \backslash E_{G}$. One shows now that the fibre $Y_{p}$ for a point $p \in Z_{G}$ coincides with the fibre $\tilde{X}_{p}=E_{G, p}$. Such a point has as its first component $A=\left(\begin{array}{ll}x & 0 \\ 0 & x\end{array}\right)$, 
and (ii) implies that its second component is of the form $[x \wedge u, x \wedge w, x \wedge v]$. Consider then the 1-parameter family $A(t)=\left(\begin{array}{cc}x & -t v \\ t u & x+t w\end{array}\right)$. For $t \neq 0,[A(t)] \in X \backslash Z_{G}$, and its lift to $\tilde{X}$ has the limit $\tilde{A}$ with components $[A]$ and $[x \wedge u, x \wedge w, x \wedge v]$. This proves (a) and also (b) as a corollary. For (c) smoothness follows from that of $X$ and $Z_{G}$, and the equivariance directly from (a).

It follows from (b) that $E_{G}$ is the $\mathbb{P}_{5}$-bundle

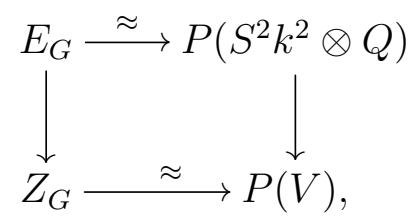

where $Q$ is the tautological quotient bundle of $\mathrm{P}(\mathrm{V})$.

Stability in $\tilde{X}$ : By definition of $\tilde{X}$ there is the Plücker embedding

$$
\tilde{X} \subset P\left(\left(\wedge^{2} k^{2} \otimes S V \oplus S^{2} k^{2} \otimes \wedge^{2} V\right) \otimes\left(S^{2} k^{2} \otimes \wedge^{2} V\right)\right)
$$

and by this the action on $\tilde{X}$ is induced by the obvious linear action of $G$ on the ambient projective space.

4.2. Proposition: Let $\tilde{A}=([A],[\xi, \omega, \eta])$ be a point of $\tilde{X}$. Then

(i) $\tilde{A}$ is semistable if and only if both of $\operatorname{det}(A)=x y^{\prime}-x^{\prime} y$ and $\omega^{2}-4 \xi \eta$ are non-zero.

(ii) If $\tilde{A} \notin E_{G}$, then $\tilde{A}$ is stable if and only if $\pi(\tilde{A})=[A]$ is stable.

(iii) If $\tilde{A} \in E_{G}$, then $\tilde{A}$ is stable if and only if $\omega^{2}-4 \xi \eta$ is not a square in $S^{2}(V / k . x)$

For the proof, notice first that the quadratic forms $\operatorname{det}(A)=x y^{\prime}-x^{\prime} y$ and $\omega^{2}-4 \xi \eta$ of the components of $\tilde{A}$ are invariant under this action. Then the statements can be canonically verified by either looking for the points in the affine cone or by using the Mumford criterion for the action of 1-parameter subgroups. For the latter, the weights can be computed via the tensor products in the Plücker space.

Some elemntary calculations with the explicit description of the group action show:

4.3. Lemma: Let $\tilde{A}=([A],[\xi, \omega, \eta])$ be a point of $\tilde{X}$. Then

(i) $\omega^{2}-4 \xi \eta=0$ if and only if there is a $g \in G$ such that $[\xi, \omega, \eta] S^{2} g=\left[\xi^{\prime}, 0,0\right]$.

(ii) $\omega^{2}-4 \xi \eta$ is square if and only if there is a $g \in G$ such that $[\xi, \omega, \eta] S^{2} g=\left[\xi^{\prime}, \omega^{\prime}, \eta^{\prime}\right]$ with $\xi^{\prime}=0$ or $\eta^{\prime}=0$.

(iii) $\omega^{2}-4 \xi \eta$ is a product if and only if there is a $g \in G$ such that $[\xi, \omega, \eta] S^{2} g=$ $\left[\xi^{\prime}, 0, \eta^{\prime}\right]$.

Let now $H_{0}^{s s} \subset H_{1}^{s s} \subset X^{s s}$ be the subvarities of points $[A]$ for which $\operatorname{det}(A)$ is a square respectively a product in $S^{2} V$. These are the inverse images in $X^{s s}$ of the double lines respectively pairs of lines in the space $P\left(S^{2} V\right)$ of conics in $P\left(V^{*}\right)$. Let $H_{0} \subset H_{1}$ be their closures in $X$. By definition $Z_{G} \subset H_{0}^{s s}$. Since the matrices $[A] \in H_{0}^{s s}$ are of type $\left[\left(\begin{array}{ll}x & 0 \\ z & x\end{array}\right)\right] g, g \in G$, one finds that $H_{0}^{s s} \backslash Z_{G}$ consists of all non-closed orbits whose closures meet $Z_{G}$, the orbits of the latter being its points. Then

$$
P(V) \cong Z_{G}=H_{0}^{s s} / / G \subset X^{s s} / / G \cong P\left(S^{2} V\right)
$$


is the Veronese embedding. Moreover, all the points $H_{0}^{s s} \backslash Z_{G}$ become unstable in $\tilde{X}$, see Lemma 4.4 .

Let denote $\tilde{H}_{0} \subset \tilde{H}_{1}$ be the proper transforms of $H_{0} \subset H_{1}$ in $\tilde{X}$. Then the following holds.

4.4. Lemma: (a) $\tilde{H}_{0} \cap \tilde{X}^{s s}=\emptyset$ and $E_{G} \cap \tilde{H}_{0}=E_{G} \backslash E_{G}^{s s}$.

(b) $\tilde{H}_{1} \cap \tilde{X}^{s}=\emptyset$ and $E_{G} \cap \tilde{H}_{1}=E_{G} \backslash E_{G}^{s}$.

Sketch of proof: A point in $H_{0}^{s s} \backslash Z_{G}$ is equivalent to a point $\left[\left(\begin{array}{ll}x & 0 \\ z & x\end{array}\right)\right]$ and this has the second component $[x \wedge z, 0,0]$ in $\tilde{X}$. By Remark 4.3 it is not semistable. Then also $\omega^{2}-4 \xi \eta=0$ for the limit points. To show that $E_{G} \backslash E_{G}^{s s} \subset \tilde{H}_{0}$ we may assume that a point $p$ in $E_{G} \backslash E_{G}^{s s}$ has the components $\left[\left(\begin{array}{ll}x & 0 \\ 0 & x\end{array}\right)\right],[x \wedge z, 0,0]$. As in the proof of Lemma 4.1, the family defined by $\left[\left(\begin{array}{cc}x & 0 \\ t z & x\end{array}\right)\right]$ shows that $p \in \tilde{H}_{0}$. This proves (a). The proof of (b) is analogous.

By the characterization of semistable points, the equivariant morphism $\pi: \tilde{X} \rightarrow X$ maps (semi-)stable points to (semi-)stable and gives rise to a morphism $\tilde{X}^{s s} / / G \rightarrow X^{s s} / / G \cong$ $P\left(S^{2} V\right)$, which is an isomorphism over the complement of the Veronese surface $Z_{G} / G$. Because $\Sigma_{2}:=E_{G}^{s s} / / G$ becomes the inverse image of $Z_{G} / G$ and is a divisor, we obtain the

4.5. Proposition: $\widetilde{P\left(S^{2} V\right)}:=\tilde{X}^{s s} / / G$ is the blow-up of $P\left(S^{2} V\right)$ along the Veronese surface.

4.6. Related geometry of conics: For any point $\tilde{A}$ in $\tilde{X}$ the quadratic form $\omega^{2}-4 \xi \eta$ can be seen as an element of $S^{2} V^{*}$ because of $\wedge^{2} V \cong V^{*}$. One can then easily verify that for any non-degenerate $A=\left(\begin{array}{ll}x & x^{\prime} \\ y & y^{\prime}\end{array}\right)\left(\right.$ for which $\left.\left.\left[x \wedge y, x \wedge y^{\prime}+x^{\prime} \wedge y, x^{\prime} \wedge y^{\prime}\right)\right]=[\xi, \omega, \eta]\right)$ ), the quadradic form $\omega^{2}-4 \xi \eta$ is the equation of the dual conic in $P(V)$ of the conic $\{\operatorname{det}(A)=0\} \subset P\left(V^{*}\right)$ (of jumping lines of the corresponding vector bundle). Because $\tilde{X}^{s s}$ is defined by $\operatorname{det}(A) \neq 0$ and $\omega^{2}-4 \xi \eta \neq 0$ we can define the universal family of conics

$$
Q \subset \tilde{X}^{s s} \times P(V)
$$

as the subvariety of pairs $(\tilde{A},[v])$ with $\left(\omega^{2}-4 \xi \eta\right)(v)=0$. If $\tilde{A} \in E_{G}^{s s}$, i.e. $A=\left(\begin{array}{ll}x & 0 \\ 0 & x\end{array}\right)$ then $(\xi, \omega, \eta)=(x \wedge u, x \wedge w, x \wedge v)$ and the fibre $Q_{\tilde{A}}$ is a pair of lines through $[\mathrm{x}]$ in $P(V)$ or a double line.

Secondly, the related quadratic form $w^{2}-4 u v \in S^{2} V / k . x$ without the factor $x$ defines two points or a double point on the double line $\left\{x^{2}=0\right\}$ in $P\left(V^{*}\right)$.

Recalling that the space of complete conics in the plane $P\left(V^{*}\right)$ consists of conics, for which the double lines are enriched by two points or a double point, one finds that $\tilde{X}^{s s}$ parametrizes this space and that the quotient $\widehat{P\left(S^{2} V\right)}:=\tilde{X}^{s s} / / G$ is the space of complete conics in $P\left(V^{*}\right)$. Moreover, because the forms $\operatorname{det}(A)$ and $\omega^{2}-4 \xi \eta$ are invariant, the conic bundle $\mathrm{Q}$ descents to a conic bundle embedded in $\widetilde{P\left(S^{2} V\right)} \times P(V)$ and describes the duality for complete conics.

\section{KirWan BlOW-UP II}

It is easy to see that there are no semistable points in $\tilde{X}$ with a 2-dimensional stabilizer by checking the types of points. But there are 1-dimensional such stabilizers. For the Kirwan blow-up it is enough to consider only connected reductive ones. Again by checking the 
different types of points, one finds that the only such stabilizers are $R=\left\{\left(\begin{array}{cc}\alpha & 0 \\ 0 & \alpha^{-1}\end{array}\right)\right\} \cong k^{*}$ and its conjugates. According to Ki we consider for the center of the blow-up of $\tilde{X}$ the subvariety $Z_{R}$ of points $\tilde{A}$ in $\tilde{X}$ which are fixed by $R$ and such in addition $R$ acts trivially on the affine fibre of $\tilde{A}$ in $\wedge^{2}\left(k^{2} \otimes V\right) \otimes\left(S^{2} k^{2} \otimes \wedge^{2} V\right)$. A direct computation shows that

$Z_{R}$ is the set of points $\left(\left[\left(\begin{array}{ll}x & 0 \\ 0 & y\end{array}\right)\right],[0, \omega, 0]\right)$ in $\tilde{X}$.

Then $G Z_{R} \subset \tilde{X}^{s s} \backslash \tilde{X}^{s}$ and is of dimension 6 .

By definition $G Z_{R} \subset \tilde{H}_{1}^{s s}$, and $G Z_{R}$ is the subset of points in $\tilde{H}_{1}^{s s}$ with closed orbits. The good quotient $G Z_{R} / / G=\tilde{H}_{1}^{s s} / / G$ is then the proper transform in $\widetilde{P\left(S^{2} V\right)}$ of the divisor $\Sigma_{1}$ of products in $P\left(S^{2} V\right)$.

\subsection{Lemma:}

(1) The closure $\overline{G Z}_{R}$ is the subvariety of points $([A],[\xi, \omega, \eta])$ in $\tilde{X}$ for which $\xi, \omega, \eta$ are pairwise linearly dependent in $\wedge^{2} V$.

(2) $\overline{G Z}_{R} \cap \tilde{X}^{s s}=G Z_{R}$.

(3) $\overline{G Z}_{R}$ is smooth.

(4) $\overline{G Z}_{R}$ and $E_{G}$ intersect transversily in dimension 5.

Proof. Let $Y$ be the closed subvariety of $\tilde{X}$ defined by the condition in (1). Then $\overline{G Z}_{R} \subset Y$. When $y \in Y \cap \tilde{X}^{s s}$, then $y=([A],[a \xi, b \xi, c \xi])$ with $b^{2}-4 a c \neq 0$ and there is a group element $g$ and some $\lambda$ so that $\lambda(a, b, c)=(0,1,0) S^{2} g$, because $y$ is supposed to be semistable. Then $y g^{-1}=([B],[0, \xi, 0])$ and thus an element of $Z_{R}$. Now $Y \cap \tilde{X}^{s s}=G Z_{R}$. If $y$ is unstable, there is a group element $g$ so that $\lambda(a, b, c)=(1,0,0) S^{2} g$. Then $y g^{-1}=$ $([B],[\xi, 0,0])$ and such points are limits of points in $G Z_{R}$ : such matrices $B$ can only be of type $\left[\left(\begin{array}{ll}x & 0 \\ y & 0\end{array}\right)\right]$ or of type $\left[\left(\begin{array}{ll}x & 0 \\ y & x\end{array}\right)\right]$. In the first case $\left[\left(\begin{array}{ll}x & 0 \\ y & t y\end{array}\right)\right]$ is family, whose members are G-equivalent to points in $Z_{R}$ for $t \neq 0$. In the second case the members of the family $\left[\left(\begin{array}{cc}x & t^{2} y \\ y & x\end{array}\right)\right]$ for $t \neq 0$ are G-equivalent to $\left[\left(\begin{array}{cc}x+t y & 0 \\ 0 & x-t y\end{array}\right)\right]$ belonging also to $Z_{R}$. This proves $Y \subset \overline{G Z}_{R}$ and thus (1) and (2). The lengthy but elementary proof of (3) and (4) by use of local coordinates for the Grassmannian and its blow-up is omitted here.

Remark: The set $\overline{G Z}_{R} \backslash G Z_{R}$ consists entirely of the orbits of the unstable points $\left(\left[\left(\begin{array}{ll}x & 0 \\ y & 0\end{array}\right)\right],[x \wedge y, 0,0]\right)$ and $\left(\left[\left(\begin{array}{ll}x & 0 \\ y & x\end{array}\right)\right],[\xi, 0,0]\right)$.

5.2. Lemma: $E_{G} \backslash E_{G}^{s s} \subset E_{G} \cap \overline{G Z}_{R} \subset E_{G} \cap \tilde{H}_{1}=E_{G} \backslash E_{G}^{s}$ and these sets are of dimension 4,5,6 respectively.

Proof. When a point $p \in E_{G}$ is unstable, it is in the orbit of a point $q=\left(\left[\left(\begin{array}{cc}x & 0 \\ 0 & x\end{array}\right)\right],[\xi, 0,0]\right)$ and then $p \in \overline{G Z}_{R}$. Such points have a 2-dimensional stabilizer $G_{q}$ and then $E_{G} \backslash E_{G}^{s s}$ is parametrized by $P(Q) \times G / G_{q}$, where $Q$ is the tautological quotient bundle on $P(V)$. Hence $E_{G} \backslash E_{G}^{s s}$ is 4-dimensional. The points in $E_{G} \cap \overline{G Z}_{R}$ are of type $\left(\left[\left(\begin{array}{cc}x & 0 \\ 0 & x\end{array}\right)\right],[a \xi, b \xi, c \xi],\right)$ with $\xi=x \wedge u$ and $u \in V / k$.x. Therefore there is a surjective morphism $P(Q) \times \mathbb{P}_{2} \rightarrow$ $E_{G} \cap \overline{G Z}_{R}$ which is generically injective. Hence $\operatorname{dim}\left(E_{G} \cap \overline{G Z}_{R}\right)=5$. Finally $E_{G} \cap \tilde{H}_{1}$ is an intersection of hypersurfaces and so of dimension 6 . 
The condition in Lemma 5.1 for points in $\overline{G Z}_{R}$ is equivalent to the vanishing of $\xi \wedge \omega, \xi \wedge \eta, \omega \wedge \eta$. Moreover, the homomorphism $(\xi, \omega, \eta) \mapsto(\xi \wedge \omega, \xi \wedge \eta, \omega \wedge \eta)$ describes the canonical wedge map

$$
\operatorname{Hom}\left(\left(S^{2} k^{2}\right)^{*}, \wedge^{2} V\right) \rightarrow \operatorname{Hom}\left(\wedge^{2}\left(S^{2} k^{2}\right)^{*}, \wedge^{2} \wedge^{2} V\right),
$$

and this is $G$-equivariant, explicitly described by

$$
(\xi, \omega, \eta)\left(\begin{array}{ccc}
\alpha^{2} & 2 \alpha \beta & \beta^{2} \\
\alpha \gamma & \alpha \delta+\beta \gamma & \beta \delta \\
\gamma^{2} & 2 \gamma \delta & \delta^{2}
\end{array}\right) \mapsto(\xi \wedge \omega, \xi \wedge \eta, \omega \wedge \eta)\left(\begin{array}{ccc}
\alpha^{2} & \alpha \beta & \beta^{2} \\
2 \alpha \gamma & \alpha \delta+\beta \gamma & 2 \beta \delta \\
\gamma^{2} & \gamma \delta & \delta^{2}
\end{array}\right) .
$$

So the map

$$
\tilde{X} \backslash \overline{G Z}_{R} \stackrel{\Phi}{\longrightarrow} P\left(\wedge^{2}\left(S^{2} k^{2}\right) \otimes \wedge^{2} \wedge^{2} V\right) \cong P\left(k^{3} \otimes V\right),
$$

given by $p \rightarrow[\xi \wedge \omega, \xi \wedge \eta, \omega \wedge \eta]$ is well-defined and $G$-equivariant and the components of this map generate the ideal sheaf of $\overline{G Z}_{R}$

The second Kirwan blow-up can now be defined as the blow-up of $\tilde{X}$ along $\overline{G Z}_{R}$ :

$$
Y:=B l_{\overline{G Z}_{R}}(\tilde{X}) \stackrel{\pi}{\longrightarrow} \tilde{X}
$$

It is simultanously the closure of the graph of $\Phi$. By the smoothness of the ingridients, $Y$ is smooth. Moreover, $Y \subset \tilde{X} \times P\left(k^{3} \otimes V\right)$ is acted on by $G$ and the projection $Y \rightarrow \tilde{X}$ is $G$-equivariant according to formula (3). We let $E_{R}$ denote the exeptional divisor.

Remark: The condition for $\overline{G Z}_{R}$ says that the second components of its points are of type $[a \xi, b \xi, c \xi]=(a, b, c) \otimes \xi$ in $P\left(S^{2} k^{2} \otimes \wedge^{2} V\right)$. This means that $\overline{G Z}_{R}$ is the pull back of the Segre variety $S=P\left(S^{2} k^{2}\right) \times P\left(\wedge^{2} V\right)$ in $P\left(S^{2} k^{2} \otimes \wedge^{2} V\right)$. It follows that also the blow-up $B l_{\overline{G Z}}(\tilde{X})$ is the pull back of the blow-up of $P\left(S^{2} k^{2} \otimes \wedge^{2} V\right)$ along the Segre variety $S$.

5.3. Stability in $Y$ : By definition $Y$ is embedded in $\tilde{X} \times P\left(\wedge^{2}\left(S^{2} k^{2}\right) \otimes \wedge^{2} \wedge^{2} V\right)$. Combined with Segre embeddings we have

$$
Y \subset P\left(\left(\wedge^{2} k^{2} \otimes S^{2} V \oplus S^{2} k^{2} \otimes \wedge^{2} V\right) \otimes\left(S^{2} k^{2} \otimes \wedge^{2} V\right) \otimes\left(\wedge^{2}\left(S^{2} k^{2}\right) \otimes \wedge^{2} \wedge^{2} V\right)\right) .
$$

Then using the Mumford criterion and considering the weights of 1-parameter subgroups one can derive:

(i) Points in $Y$ over points in $G Z_{R}$ are stable.

(ii) Points in $Y$ over stable points in $\tilde{X}$ are stable.

(iii) Points in $Y$ over unstable points in $\tilde{X}$ are unstable.

(iv) Properly semistable points in $\tilde{X}^{s s} \backslash G Z_{R}$ become unstable in $Y$.

(v) Every semistable point in $Y$ is stable.

Remark: One can as well show that the stabilizer of any semistable point in $Y$ is finite.

The $G$-equivariant morphism $\pi$ induces a surjective $G$-equivariant morphism $Y^{s} \longrightarrow \tilde{X}^{s s}$ and thus a surjective morphism of the good quotients

$$
\tau: Y^{s} / G \longrightarrow \tilde{X}^{s s} / / G=\widetilde{P\left(S^{2} V\right)}
$$

with surjective restriction

$$
\tilde{\Sigma}_{1}:=E_{R}^{s} / G \longrightarrow G Z_{R} / / G=\Sigma_{1},
$$


whereas

$$
Y^{s} / G \backslash \tilde{\Sigma}_{1} \stackrel{\approx}{\longrightarrow} \tilde{X}^{s s} / / G \backslash \Sigma_{1}
$$

must be an isomorphism because $\pi$ is an isomorphism outside $E_{R}$. Moreover, because $Y^{s} \stackrel{\pi}{\longrightarrow} \tilde{X}^{s s}$ is a blow up, also the induced morphism $\tau$ is a blow-up along the divisor $\Sigma_{1}$. Hence the

5.4. Proposition: $\tau: Y^{s} / G \longrightarrow \tilde{X}^{s s} / / G=\widetilde{P\left(S^{2} V\right)}$ is an isomorphism.

Remark: While the second Kirwan blow-up has no effect on the quotient, it describes $\widehat{P\left(S^{2} V\right)}$ as a geometric quotient, so that non-isomorphic S-equivalent limit sheaves w.r.t. the parameter space $Y^{s}$ are excluded. This is needed for the construction of families which include admissible tree bundles because S-equivalence for tree bundles is not defined.

\section{FAMilies INCLUDing TREE BUNDLES}

In this section the construction of families of sheaves, including all admissible tree bundles for the tree compactification of $M^{b}(0,2)$, will be sketched in two steps. In step one we construct such a family over the base space $\tilde{X}^{s s}$.

Firstly we recall the presentation of the semi-universal family for the Gieseker-Maruyama space $M(2 ; 0,2)$. Let $0 \rightarrow \mathcal{U} \rightarrow k^{2} \otimes \mathcal{O}_{X} \rightarrow \mathcal{Q} \rightarrow 0$ be the tautological sequence on the Grassmannian $X=G_{2}\left(k^{2} \otimes V\right)$. As in formula (1) there are two such equivalent presentations. The second is the exact sequence over $X^{s s} \times P$

$$
0 \rightarrow k^{2} \otimes \mathcal{O}_{X} \otimes \mathcal{O}_{P}(-2) \longrightarrow \mathcal{Q} \otimes \mathcal{O}_{P}(-1) \rightarrow \mathcal{F} \rightarrow 0
$$

Recall from 4.3 that $H_{1}^{s s} \subset X^{s s}$ is the hypersurface of points $[A]$ for which $\operatorname{det}(A) \operatorname{decom-}$ poses, i.e. the inverse image of $\Sigma_{1}$, and that $H_{0}^{s s} \subset H_{1}^{s s}$ is the subvariety where $\operatorname{det}(A)$ is a square. Let now $S_{1} \subset X^{s s} \times P$ be the subvariety of points $([A],[v])$ for which $v$ divides $\operatorname{det}(A)$, and $S_{0} \subset S_{1}$ where $\operatorname{det}(A)=v^{2}$. Then $S_{1}$ is 7 -dimensional and 2:1 over $H_{1}^{s s} \backslash H_{0}^{s s}$. It follows that $\mathcal{F}$ is locally free on $X^{s s} \times P \backslash S_{1}$ whose restriction to fibres over $X^{s s} \backslash H_{1}$ are the vector bundles in $M^{b}(0,2)$, whereas the sheaves over points in $H_{1}$ become the semistable sheaves in the boundary of $M^{b}(0,2)$.

Notice however that the sheaf $\mathcal{F}$ restricted to $\{p\} \times P$ may be singular only in one of the points of $S_{1}$ over $p$, see the Notice before 3.1 .

\subsection{First step:}

Let now $\tilde{X}^{s s} \times P \stackrel{\alpha}{\rightarrow} X^{s s} \times P$ be the map $\phi=\alpha \times$ id, where $\alpha$ is the blow-up map of section 4 , and consider the lifted family $\mathbb{F}=\phi^{*} \mathcal{F}$. Then $\mathbb{F}$ is locally free over the inverse image of $X^{s s} \backslash E_{G}^{s s} \cup \tilde{H}_{1}^{s s}$

Analogously to $S_{0}$ and $S_{1}$, let then $\tilde{S}_{0}$ the set of points $(p,[v]) \in \tilde{X}^{s s} \times P$ over $E_{G}^{s s}$ where $\operatorname{det}(A)=v^{2}$, and let similarly $\tilde{S}_{1} \subset \tilde{X}^{s s} \times P$ be the set of points over $\tilde{H}_{1}^{s s}$ where $v$ is a factor of $\operatorname{det}(A)$. Then $\mathbb{F}$ is locally free outside $\tilde{S}_{0} \cup \tilde{S}_{1}, \tilde{S}_{0}$ is mapped 1:1 to $E_{G}^{s s}$ and the $\operatorname{map} \tilde{S}_{1} \backslash \tilde{S}_{0} \rightarrow \tilde{H}_{1}^{s s} \backslash E_{G}^{s s}$ is $2: 1$.

Consider now the blow-up $Z \stackrel{\sigma_{0}}{\longrightarrow} \tilde{X}^{s s} \times P$ along $\tilde{S}_{0}$ and let $D$ denote the exceptional divisor. Let

$$
\mathcal{F}:=\sigma_{0}^{*} \mathbb{F} / \text { torsion }
$$


be the torsion free pullback on $Z$. Now the situation of the families $\mathcal{F}$ and $\mathbb{F}$ restricted to the open subset $\tilde{X}^{s s} \backslash \tilde{H}_{1}^{s s}$ of the base is the higher dimensional analog to that of the families over the curve $C$ in section 3.1 , with $0 \in C$ replaced by the divisor $E_{G}^{s s} \subset$ $\tilde{X}^{s s} \backslash \tilde{H}_{1}^{s s}$.

Moreover, one can compare the two situations by considering a curve $C \subset \tilde{X}^{s s}$ transversal to $E_{G}^{s s}$ in a point $p \notin \tilde{H}_{1}^{s s}$. Then the blow-up $Z_{C}$ of $C \times P$ in the point $(p, q) \in \tilde{S}_{0}$ can be identified with the restriction of $Z$ to $C$. Moreover, by flatness, the sheaves $\mathcal{F}_{C}$ and $\mathbb{F}_{C}$ on $Z_{C}$ from 3.1 can be identified with the restrictions of $\mathcal{F}$ and $\mathbb{F}$ to $Z \mid C$. Because $\mathcal{F}_{C}$ is locally free on $Z_{C}$, it follows that $\mathcal{F}$ is locally free in a neighborhood of the fibre $Z_{p}$ of $Z$ over $p$. Finally, because the fibre $Z_{p}$ is the union of the blow-up of $P$ at $q$ and the restriction $D_{p}$ of exceptional divisor $D$, the sheaf $\mathcal{F} \mid Z_{p}$ is a tree bundle on $Z_{p}$. In order to obtain the correct Chern classes, we have to replace $\mathcal{F}$ by its twist $\mathcal{F}(D)$ as in 3.1 . which is also compatible with the restriction. It has been shown:

6.1.1. Proposition: With the notation above, the family $\mathcal{F}$ is a family of tree bundles over the restricted base variety $\tilde{X}^{s s} \backslash \tilde{H}_{1}^{s s}$.

If $p \in \tilde{X}^{s s} \backslash \tilde{H}_{1}^{s s} \cup E_{G}^{s s}$, then $\mathcal{F} \mid Z_{p}, Z_{p}=P$, is a bundle in $M^{b}(0,2)$.

If $p \in E_{G}^{s s} \backslash \tilde{H}_{1}^{s s}$, then $\mathcal{F} \mid Z_{p}$, where $Z_{p}=\tilde{P} \cup D_{p}, D_{p} \cong \mathbb{P}_{2}$, is a tree bundle with $\mathcal{F} \mid \tilde{P} \cong 2 \mathcal{O}_{\tilde{P}}$ and $\mathcal{F} \mid D_{p} \in M_{D_{p}}^{b}(0,2)$.

For the fibres over points in $\tilde{H}_{1}^{s s}$ we have:

6.1.2. Lemma: Let $\hat{S}_{1}$ be the proper transform of $\tilde{S}_{1}$ in $Z$. Then $\hat{S}_{1} \rightarrow \tilde{H}_{1}^{s s}$ is $2: 1$.

Remark: For a point $p \in E_{G}^{s s} \cap \tilde{H}_{1}^{s s}$ the two points of $\hat{S}_{1}$ over $p$ will be contained in the fibre $D_{p} \cong \mathbb{P}_{2}$ of the exceptional divisor $D$. By the previous, $\mathcal{F}$ is locally free on $Z \backslash \hat{S}_{1}$.

Proof. The method of proof is again by restriction to transversal curves: Let $q \in \tilde{S}_{0} \cap \tilde{S}_{1} \subset$ $\tilde{X}^{s s} \times P$ and $p \in E_{G}^{s s} \cap \tilde{H}_{1}^{s s}$ its image. Then $p$ has the components $\left[\begin{array}{cc}x & 0 \\ 0 & x\end{array}\right]$ and $[a \xi, b \xi, c \xi]$ with $b^{2}-4 a c \neq 0$ and $\xi=x \wedge y$ for some $y \in V$. Then

$$
p(t):=\left(\left[\begin{array}{cc}
x & 0 \\
0 & x+t y
\end{array}\right],[a \xi, b \xi, c \xi]\right)
$$

is a 1-parameter family in $G Z_{R} \subset \tilde{H}_{1}^{s s}$ defining a normal direction to $E_{G}^{s s}$ at $p$. Let $C$ denote the image of $p(t)$ for small $t$. For $t \neq 0$ the points $[\mathrm{x}]$ and $[\mathrm{x}+$ ty] define then sections of $\tilde{S}_{1} \mid C \backslash\{0\}$, which fill this subset. Because $q$ is the only point in $\tilde{S}_{0}$ over $p, q \in \tilde{S}_{1} \mid C$, the closure of $\tilde{S}_{1} \mid C \backslash\{0\}$. Let now $S_{C}:=\tilde{S}_{1} \mid C \subset C \times P$ and consider the blow-ups

$$
B l_{q}(C \times P) \subset B l_{\tilde{S}_{0}}\left(\tilde{X}^{s s}\right)=Z
$$

as the proper transform. Then the restriction $\hat{S}_{1} \mid C$ of the proper transform $\hat{S}_{1}$ can be identified with the proper transform of $S_{C}$ in $B l_{q}(C \times P)$. This situation corresponds to the figure in 3.1 with the two sections $[\mathrm{x}]$ and $[\mathrm{x}+\mathrm{ty}]$ added. Then the proper transforms of these linear sections do not meet on the exceptional divisor $D_{p}$. Hence also $\hat{S}_{1} \cap D_{p}$ consists of two different points.

\subsection{Second step:}

By the above, $\mathcal{F}$ is locally free on $Z \backslash \hat{S}_{1}$ and one could try to construct the tree bundles over $\tilde{H}_{1}^{s s}$ by directly blowing up $Z$ along $\hat{S}_{1}$ and modifying the lifted sheaf. However, 
over points $p \in \tilde{H}_{1}^{s s} \backslash G Z_{R}$ the sheaf $\mathcal{F} \mid Z_{p}$ has only one singular point and is not stable, see the remark at the beginning of this section 6 . Secondly, $\tilde{H}_{1}^{s s} \backslash G Z_{R}$ consists only of non-closed orbits. On the other hand the orbits in $G Z_{R}$ are closed and for $p \in G Z_{R}$ the two points of $\hat{S}_{1}$ are the singular points of $\mathcal{F} \mid Z_{p}$.

Now this insufficiency can be eliminated by using the second Kirwan blow-up $Y \rightarrow \tilde{X}$ and pulling the pair $(Z, \mathcal{F})$ back to $Y^{s}$. After this the points of $\tilde{H}_{1}^{s s} \backslash G Z_{R}$ become unstable and can be neglected, and $\hat{S}_{1} \mid G Z_{R}$ is the reasonable locus to be blown up. Therefore, let

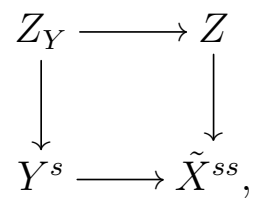

be the pull back of $Z$ and let $\mathcal{F}_{Y}$ be the lift of $\mathcal{F}$ to $Z_{Y}$. The situation of the pair $\left(Z_{Y}, \mathcal{F}_{Y}\right)$ is now the relative version of the situation in 3.2 before using a double cover.

6.2.1. Properties of $\left(Z_{Y}, \mathcal{F}_{Y}\right)$ :

Let $E_{R}^{s}$ denote the exceptional divisor of $Y^{s}$ over $G Z_{R}$, see section 5 , let $E_{G, Y}$ denote the proper transform of $E_{G}$, and let $D_{Y}$ be the pull back of $D$ in $Z$. Then $\mathcal{F}_{Y}$ is singular exactly along the pull back $S_{1, Y}$ of $\hat{S}_{1}$ and $S_{1, Y}$ is $2: 1$ over $E_{R}^{s}$ everywhere by 6.1.2. For points $p$ in $E_{R}^{s} \backslash E_{G, Y}$, the two points of $S_{1, Y}$ over $p$ will be in the fibre $Z_{Y, p} \cong P$, but for points $p$ in $E_{R}^{s} \cap E_{G, Y}$, the two points of $S_{1, Y}$ over $p$ will be in the fibre $D_{Y, p}$ of $D_{Y}$.

Remark: The variety $Z_{Y}$ may also be obtained as the blow-up of the variety $S_{0, Y} \subset Y^{s} \times P$ over $E_{G, Y}$, defined as $\tilde{S}_{0}$ over $E_{G}$.

In order to construct a family of tree bundles in this new relative situation, $Z_{Y}$ has to be blown up along $S_{1, Y}$ as in the case 3.2 . Then the torsionfree pull back of $\mathcal{F}_{Y}$ would give a family of tree bundles parametrized along $E_{R}^{s}$. But as in 3.2 these tree bundles would not be admissible as defined in 2.2. In analogy to 3.2 one would have to use a double cover of $Y^{s}$ which is branched exatly over $E_{R}^{s}$ in order to construct admissible tree bundles. However, such a double cover may not exist globally. But one could consider such local covers $U \rightarrow Y^{s}$ over affine open parts. Then we have Cartesian diagrams

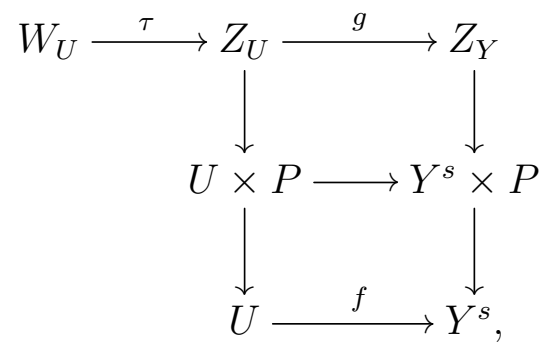

where $\tau$ is the blow-up of $Z_{U}$ along the subvariety $S_{U}=g^{*} S_{1, Y}$. This is the subvariety where $\mathcal{F}_{U}:=g^{*} \mathcal{F}_{Y}$ is not locally free. By the previous, it is $2: 1$ over the branch locus $B:=f^{*} E_{R}^{s} \subset U$. Consider then the sheaf

$$
\mathcal{E}:=\tau^{*} \mathcal{F}_{U} / \text { torsion }
$$

One can show as in the curve case that $\mathcal{E}$ is flat over $U$. 
Now one can argue as in 6.1 using curves $C$ which are transversal to $B$ : There is an elementary transform $\mathcal{E}^{\prime}$ of $\mathcal{E}$ on $W_{U}$ with transformation support over $B$ which is locally free on $W_{U}$. Then $\mathcal{E}^{\prime}$ is a family of tree bundles, whose fibres over points in $U \backslash B$ are the same as for points in $Y^{s} \backslash E_{R}^{s}$ or in $\tilde{X}^{s s} \backslash G Z_{R}$. After twisting with the exceptional divisor in $W_{U}$, we may finally assume that $\mathcal{E}^{\prime}$ is a family of admissible tree bundles with prescribed Chern classes. Hence the

6.2.2. Proposition: For any 2:1 cover $U \stackrel{f}{\rightarrow} Y^{s}$ of an affine open subset of $Y^{s}$, branched exactly along $E_{R}^{s}$, the following holds:

(i) For points $p$ in $U \backslash f^{*} \tilde{E}_{G} \cup B$ the bundle $\mathcal{E}_{p}^{\prime}$ is a member of $M_{W_{U, p}}^{b}(0,2)$, where $W_{U, p} \cong \mathbb{P}_{2}$.

(ii) For points $p$ in $f^{*} \tilde{E}_{G} \backslash B$ the bundle $\mathcal{E}_{p}^{\prime}$ is of the type described in 3.1.

(iii) For points $p$ in $B \backslash f^{*} \tilde{E}_{G}$ the bundle $\mathcal{E}_{p}^{\prime}$ is of the type described in 3.2.

(iv) For points $p$ in $B \cap f^{*} \tilde{E}_{G}$ the bundle $\mathcal{E}_{p}^{\prime}$ is of the type described in 3.3.

The families of tree bundles so constructed may not descend to a global family over the Kirwan blow-up $\tilde{M}_{2} \cong \widehat{P\left(S^{2} V\right)}$ of $M(2 ; 0,2) \cong P\left(S^{2} V\right)$ because the automorphism groups of the tree bundles include automorphisms of the supporting surfaces, see 2.2. However, delicately, their isomorphism classes are determined precisely by the points of $\tilde{M}_{2}$ :

6.2.3. Proposition: The set of points of $\tilde{M}_{2}$ is the set isomorphism classes of the tree bundles constructed above. In particular, let as above $\Sigma_{2} \subset \widehat{P\left(S^{2} V\right)}$ be the blow-up of the Veronese surface in $P\left(S^{2} V\right)$ and $\Sigma_{1} \subset \widehat{P\left(S^{2} V\right)}$ the proper transform of the subvariety of decomposable conics. Then

(i) $\tilde{M}_{2} \backslash \Sigma_{1} \cup \Sigma_{2}=M^{b}(0,2)$ is the set the isomorphism classes of the (stable) bundles in $M(2 ; 0,2)$.

(ii) The set $\Sigma_{2} \backslash \Sigma_{1}$ is the set of isomorphism classes of limit tree bundles of type 1 described in 3.1 .

iii) The set $\Sigma_{1} \backslash \Sigma_{2}$ is the set of isomorphism classes of limit tree bundles of type 2 described in 3.2.

(iv) The set $\Sigma_{1} \cap \Sigma_{2}$ is the set of isomorphism classes of limit tree bundles of type 3 described in 3.3 .

Proof. There is nothing to proof for (i). For the proof of (ii), recall that $\Sigma_{2} \backslash \Sigma_{1}$ is the geometric quotient of the open part $E_{G}^{s} \subset \tilde{X}^{s}$ of the exceptional divisor $E_{G}$ whose points are of type

$$
p=\left(\left[\left(\begin{array}{cc}
x & 0 \\
0 & x
\end{array}\right)\right],[\xi, \omega, \eta]\right),
$$

where $\omega^{2}-4 \xi \eta$ decomposes into two different factors and $\xi, \omega, \eta \in x \wedge V$. By 4.3 we may assume that $\omega=0$. The two factors $\xi, \eta$ determine two lines in $\mathrm{P}=\mathrm{P}(\mathrm{V})$ through $[x]$, see 4.6. Now the fibre $Z_{p}$ is a union $\tilde{P}(x) \cup D_{p}$, where $\tilde{P}(x)$ is the blow-up of $P$ at $[x]$ and $D_{p} \cong \mathbb{P}_{2}$. Then the two lines in $P$ determine two points $q_{1}, q_{2}$ on the exceptional line $\ell_{p}=\tilde{P}(x) \cap D_{p}$. Let now $\mathcal{F}$ on $Z$ be the sheaf constructed in 6.1. By 6.1.1 $\mathcal{F} \mid Z_{p}$ has the restrictions $\mathcal{F} \mid \tilde{P}(x) \cong 2 \mathcal{O}_{\tilde{P}(x)}$ and $\mathcal{F} \mid D_{p} \in M_{D_{p}}^{b}(0,2)$. So $\mathcal{F} \mid D$ corresponds to its smooth conic of jumping lines in the dual plane $D_{p}^{*}$ or to the dual conic $\Gamma_{p} \subset D_{p}$ of the latter.

Claim: The conic $\Gamma_{p}$ meets the line $\ell_{p}$ in the two points $q_{1}, q_{2}$. 
In addition, there is the following elementary

6.2.4. Lemma: Let $\ell$ be the line through two points $a_{1}, a_{2} \in \mathbb{P}_{2}$ and let $\mathrm{Aut}_{\ell}\left(\mathbb{P}_{2}\right)$ be the subgroup of the group of automorphisms of $\mathbb{P}_{2}$ which fixes the points of $\ell$. Then Aut ${ }_{\ell}\left(\mathbb{P}_{2}\right)$ acts transitively on the set of non-degenerate conics through $a_{1}, a_{2}$.

If the claim is verified, the Lemma implies that the isomorphism class of $\mathcal{F} \mid D_{p}$ and then also of $\mathcal{F} \mid Z_{p}$ only depends on the two points $q_{1}, q_{2}$, which are determined by the point $p$. Then the isomorphism class of $\mathcal{F} \mid Z_{p}$ also depends only on the image $[p]$ of $p$ in the quotient $\tilde{M}_{2}$, which proves (ii).

In order to prove the claim we use again 1-parameter degenerations with limit point $p$ which are transversal to $E_{G}^{s}$.

For that we may assume that

$$
p=\left(\left[\left(\begin{array}{cc}
e_{0} & 0 \\
0 & e_{0}
\end{array}\right)\right],\left[e_{0} \wedge e_{1}, 0, e_{0} \wedge e_{2}\right]\right),
$$

where $e_{0}, e_{1}, e_{2}$ form a basis of $V$, and that the first component of the 1-parameter family is given by

$$
A(t)=\left(\begin{array}{cc}
e_{0} & 0 \\
0 & e_{0}
\end{array}\right)+t\left(\begin{array}{ll}
x & x^{\prime} \\
y & y^{\prime}
\end{array}\right) .
$$

with $t$ in a neighborhood $C$ of $0 \in \mathbb{A}^{1}(k)$. This is a smooth curve in $X^{s s}$ and its lift to $\tilde{X}^{s s}$ is transversal to $E_{G}^{s}$ and has second component

$$
\left[e_{0} \wedge y+t \xi, e_{0} \wedge\left(y^{\prime}-x\right)+t \omega,-e_{0} \wedge x^{\prime}+t \eta\right],
$$

where $(\xi, \omega, \eta)=\left(x \wedge y, x \wedge y^{\prime}+x^{\prime} \wedge y, x^{\prime} \wedge y^{\prime}\right)$. Because $p$ is supposed to be the limit at $t=0$, we may assume, up to a scalar factor, that the components of the vectors satisfy

$$
y_{1}=1, y_{2}=0, y_{1}^{\prime}=x_{1}, y_{2}^{\prime}=x_{2}, x_{1}^{\prime}=0, x_{2}^{\prime}=-1
$$

In addition we replace the basis $e_{1} \wedge e_{2},-e_{0} \wedge e_{2}, e_{0} \wedge e_{1}$ of $\wedge^{2} V$ by the basis $z_{0}, z_{1}, z_{2}$ of $V^{*}$, dual to the basis $e_{0}, e_{1}, e_{2}$ of $V$. Then the second component of $p(t)$ reads

$$
\left[z_{2}-t x_{2} z_{0}+t \xi^{\prime}, t z_{0}+t \omega^{\prime},-z_{1}+x_{1} z_{0}+t \eta^{\prime}\right],
$$

where $\xi^{\prime}, \omega^{\prime}, \eta^{\prime} \in \operatorname{Span}\left(z_{1}, z_{2}\right)$.

Let now $Z_{C}$ be the restriction of $Z$ to $C$. Then $Z_{C}$ can considered the blow up of $C \times P$ at $\left(0,\left[e_{0}\right]\right)$ as a proper transform and $\mathcal{F} \mid D_{p}$ can be computed as in 3.1 , as well as its conic $\Gamma_{p} \subset D_{p}$. As $\mathcal{F} \mid Z_{C}$ is the torsion free pull back of the sheaf on $C \times P$ defined by $A(t)$, its family of conics becomes the proper transform of the family

$$
Q=\left\{\left(t z_{0}+t \omega^{\prime}\right)^{2}-4\left(z_{2}-t x_{2} z_{0}+t \xi^{\prime}\right)\left(-z_{1}+t x_{1} z_{0}+t \eta^{\prime}\right)=0\right\},
$$

whose fibres for $t \neq 0$ are the conics of $\mathcal{F} \mid\{t\} \times P$, c.f. 4.6. This proper transform is obtained by substituting the forms $t z_{0}, z_{1}, z_{2}$ by $u_{0}, u_{1}, u_{2}$, which are the coordinate forms of $D_{p} \cong \mathbb{P}_{2}$, see 3.1. So the proper transform $\tilde{Q}$ of $Q$ is defined by the equation

$$
\left(u_{0}+t \omega^{\prime}\right)^{2}-4\left(u_{2}-x_{2} u_{0}+t \xi^{\prime}\right)\left(-z_{1}+x_{1} u_{0}+t \eta^{\prime}\right),
$$

where now $\xi^{\prime}, \omega^{\prime}, \eta^{\prime} \in \operatorname{Span}\left(u_{1}, u_{2}\right)$. For $t=0$ the conic $\Gamma_{p}$ of $\mathcal{F} \mid D_{p}$ has the equation $u_{0}^{2}-4\left(u_{2}-x_{2} u_{0}\right)\left(-u_{1}+x_{1} u_{0}\right)$. Because the line $\ell_{p}$ and $P\left(\left[\tilde{e}_{0}\right]\right)$ is given by $u_{0}=0, \Gamma_{p}$ meets $\ell_{p}$ in the two points $q_{1}, q_{2}$ with equation $u_{1} u_{2}$. This proves the claim and thus (ii) of proposition 6.2.3. 
For the proof of (iii) let a point in $\Sigma_{1} \backslash \Sigma_{2}$ be the image of a point $p \in E_{R}^{s} \subset Y^{s}$. We may assume that its image $\bar{p} \in G Z_{R}$ under the second blow up has the components

$$
\left(\begin{array}{cc}
x_{1} & 0 \\
0 & x_{2}
\end{array}\right),\left[0, x_{1} \wedge x_{2}, 0\right] \text {. }
$$

Under an auxiliary blow-up $W_{U}$ as in 6.2.1, the fibre $W_{U, p}$ is isomorphic to $\tilde{P}\left(x_{1}, x_{2}\right) \cup D_{1} \cup$ $D_{2}$, where $\tilde{P}\left(x_{1}, x_{2}\right)$ is the blow-up of $P$ at $x_{1}, x_{2}, D_{i} \cong \mathbb{P}_{2}$, containing the exceptional lines $\ell_{i}$ of $\tilde{P}\left(x_{1}, x_{2}\right)$. Moreover, $W_{U, p}$ is determined by the data of the point $p$ or its image in $\Sigma_{1} \backslash \Sigma_{2}$ up to isomorphism. By 6.2.2, (iii), the tree bundle $\mathcal{E}_{p}^{\prime}=\mathcal{E}^{\prime} \mid W_{U, p}$ is trivial on $\tilde{P}\left(x_{1}, x_{2}\right)$ and restricts to bundles $\mathcal{E}_{i}^{\prime}$ on $D_{i}$ with Chern classes $c_{1}=0, c_{2}=2$. By the following Lemma 6.2.5 the isomorphism class of each $\mathcal{E}_{i}^{\prime}$ corresponds uniquely to a point $q_{i} \in D_{i} \backslash \ell_{i}$. Since the group $\operatorname{Aut}_{\ell_{i}}\left(D_{i}\right)$ acts transitively on $D_{i} \backslash \ell_{i}$, see 6.2.4, these isomorphism classes are uniquely determined by $W_{U, p}$, and finally determined by the point $[p] \in \Sigma_{1} \backslash \Sigma_{2}$, because the automorphisms of $W_{U, p}$ must be identities on $\tilde{P}\left(x_{1}, x_{2}\right)$.

6.2.5. Lemma: Let $\ell \subset \mathbb{P}_{2}=P$ be a line. Then the moduli space $M_{\ell}(0,1)$ of isomorphism classes of rank-2 vector bundles on $\mathbb{P}_{2}$ which are trivial on $\ell$ with Chern classes $c_{1}=0, c_{2}=1$ can be identified with the set $\mathbb{P}_{2} \backslash \ell$.

Proof of the Lemma: Let $\ell$ have the equation $z_{0}$ and let $a=\left[a_{0}, a_{1}, a_{2}\right] \in \mathbb{P}_{2} \backslash \ell$. Let

$$
B=\left(\begin{array}{cccc}
z_{0} & z_{1} & z_{2} & 0 \\
a_{0} & a_{1} & a_{2} & z_{0}
\end{array}\right)
$$

and define $\mathcal{E}(a)$ as cokernel in the sequence

$$
0 \rightarrow \mathcal{O}_{P}(-2) \oplus \mathcal{O}_{P}(-1) \stackrel{B}{\rightarrow} 3 \mathcal{O}_{P}(-1) \oplus \mathcal{O}_{P} \rightarrow \mathcal{E}(a) \rightarrow 0 .
$$

Then the class of $\mathcal{E}(a)$ belongs to $M_{\ell}(0,1)$. Conversely, given any $\mathcal{E}$ in $M_{\ell}(0,1)$, it is well known that $\mathcal{E}$ is an elementary transform of the twisted tangent bundle $\mathcal{T}_{P}(-2)$ with exact extension sequence

$$
0 \rightarrow \mathcal{T}_{P}(-2) \rightarrow \mathcal{E} \rightarrow \mathcal{O}_{\ell} \rightarrow 0
$$

From that we get a resolution matrix $B$ of $\mathcal{E}$ as above. In that, $\left(a_{0}, a_{1}, a_{2}\right)$ represents the extension class and $\left[a_{0}, a_{1}, a_{2}\right]$ the isomorphism class of $\mathcal{E}$.

This completes the proof of (iii). The proof of (iv) is analogous to that of (iii). In this case a point $p \in B \cap f^{*} \tilde{E}_{G}$ or $p \in E_{R}^{s} \cap \tilde{E}_{G}$ over a point in in $\Sigma_{1} \cap \Sigma_{2}$ can be supposed to have as components

$$
\left[\left(\begin{array}{cc}
x & 0 \\
0 & x
\end{array}\right)\right],[a \xi, b \xi, c \xi],[u, w, v]
$$

with $\xi \in x \wedge V$ and $b^{2}-a c \neq 0$. Then $W_{U, p}$ as a fibre of the blow-up is isomorphic to $\tilde{P}([x]) \cup \tilde{D}_{0}\left(p_{1}, p_{2}\right) \cup D_{1} \cup D_{2}$, where $\tilde{D}_{0}\left(p_{1}, p_{2}\right)$ is the blow-up at two points of a plane $D_{0}$ which contains the exceptional line $\ell_{0}$ of $\tilde{P}([x])$, and where $D_{i}$ are again planes containing the two exceptional lines $\ell_{i}$ of $\tilde{D}_{0}\left(p_{1}, p_{2}\right)$. Then $W_{U, p}$ depends only on the geometry and the point $p$ up to isomorphism. Now the tree bundle $\mathcal{E}^{\prime}$ on $W_{U}$ of 6.2 .2 is trivial on $\tilde{P}([x])$ and $\tilde{D}_{0}\left(p_{1}, p_{2}\right)$, whereas $\mathcal{E}^{\prime} \mid D_{i}$ has Chern classes $c_{1}=0, c_{2}=1$. It follows again from 6.2.5 that the isomorphism classes of $\mathcal{E}^{\prime} \mid D_{i}$ are unique, and then that $\mathcal{E}^{\prime} \mid W_{U, p}$ is uniquely determined because the automorphisms of $W_{U, p}$ must be identities on the components $\tilde{P}([x])$ and $\tilde{D}_{0}\left(p_{1}, p_{2}\right)$. This proves (iv) of the proposition. 


\subsection{The stack:}

By the above construction of families of tree bundles a global family of such bundles could not be obtained. Instead, we have families of tree bundles on local 2:1 covers of the parameter space $Y^{s}$. These are forming an obvious moduli stack over the category of such open covers. It is plausible to claim that this is a Deligne-Mumford stack which is corepresented by $Y^{s} / G \cong \widetilde{P\left(S^{2} V\right)}$.

In [MTT] global families of limit tree bundles of stable rank-2 vector bundles on surfaces have been constructed by other abstract procedures, which led to algebraic spaces as moduli spaces. The question of their relation to the above stack being open at present.

\section{REFERENCES}

[EH] D.Eisenbud-J.Harris, The Geometry of Schemes, GTM 197, Springer 2001

[Hr] J.Harris, Algebraic Geometry, GTM 133, Springer 1992,

[Hsh] R.Hartshorne, Algebraic Geometry, Springer 1977

[Ki] F.Kirwan, Partial desingularisations of quotients of nonsingular varieties and their Betti numbers, Ann of Math. 122, 41-85, 1985

[MTT] D.Markushevich-A.S.Tikhomirov-G.Trautmann, Bubble tree compactification of moduli spaces of vector bundles on surfaces, Cent. Eur. J. Math. 10, 1331-1355, 2012

[M] D.Mumford, Red Book of Varieties and Schemes, Springer LNM 1385, 1988

[NT] M.S.Narasimhan-G.Trautmann, Compactification of $M_{P_{3}}(0,2)$ and Poncelet pairs of conics, Pacific J. of Math. 145, 255-365, 1990

[N] P.Newstead, Introduction to moduli problems and orbit spaces, Tata Institute Lectures 51 , 1978

Universität Kaiserslautern, Fachbereich Mathematik,

ERWIN-SCHRÖDINGER-STRASSE

D-67663 KAISERSLAUTERN

E-mail address: trm@mathematik.uni-kl.de 